\title{
Phase-Enhanced Selective Ion Ejection in an Orbitrap Mass Spectrometer
}

\author{
Qizhi Hu, R. Graham Cooks, and Robert J. Noll \\ Department of Chemistry, Purdue University, West Lafayette, Indiana, USA
}

The mass resolution achieved in selective ion isolation using resonance excitation is usually limited by the frequency resolution of the ac waveform and by unintended off-resonance excitation. A new method of phase-enhanced selective ion ejection based on broadband dipolar excitation and ion ejection applicable to the Orbitrap is described and shown to allow an isolation resolution of 28,400 . The method is calculated to be able to provide a mass resolution for ion ejection of up to 100,000. (J Am Soc Mass Spectrom 2007, 18, 980-983) () 2007 American Society for Mass Spectrometry

$\mathrm{I}$ on isolation is the primary event in tandem mass spectrometry. High mass resolution in the ion selection step provides high-quality tandem mass spectrometry and, therefore, benefits analyses of complex samples [1]. For example, only when a single isotopomer of a multiply charged protein ion is mass selected will the fragment ions be isotopically pure, in turn allowing their masses to be assigned unambiguously.

The Orbitrap, a new mass analyzer, provides high mass resolution (up to 100,000), high mass accuracy (2 ppm) [2], a mass/charge ratio range of at least 6000 [3], and a linear dynamic range of $>5000$ within a mass spectrum [4]. In the Orbitrap, the mass-to-charge ratio is obtained from the frequency of harmonic ion oscillations of the orbitally trapped ions along the axis of the electric field. This axial frequency is independent of the angular and radial motion of the ions and is determined using image current detection and fast Fourier transforms. The frequency is related to the mass-to-charge ratio $(m / z)$ by the relation $\omega=(\mathrm{kz} / \mathrm{m})^{1 / 2}[5]$.

Ion motion control experiments involving massselective ion excitation and de-excitation of trapped ions in the Orbitrap using ac dipolar waveforms have been reported recently $[6,7] .^{\circ}$ These $^{\circ}$ experiments $^{\circ} \mathrm{dem}-$ onstrate that ion axial motion (i.e., in the $z$-direction) can be controlled by applying ac waveforms to the two outer electrodes of the Orbitrap. Excitation of an ion's axial motion occurs when the ac electric field is resonant with the ion's characteristic axial frequency. If the ac waveform is applied $180^{\circ}$ out of phase with respect to ion motion, the ion can be de-excited to the center plane $(z=0)$ of the Orbitrap, re-excited to re-gain full amplitude of its harmonic motion, and finally ejected from the Orbitrap if sufficient excitation is provided. Ions can

Address reprint requests to Dr. Robert J. Noll, Chemistry Department, Purdue University, 560 Oval Drive, West Lafayette, IN 47907, USA. E-mail: rnoll@purdue.edu also be ejected from the Orbitrap directly if the excitation waveform is applied in phase with (i.e., $0^{\circ}$ ) the ion motion.

The capability to control ion axial motion in the Orbitrap is important because it provides the possibility of improving the performance of the Orbitrap and adding new functions to this device. For example, ac excitation and ejection can be used to explore interpacket space charge effects in the Orbitrap. The evaluation of space charge (ion-ion repulsion) effects either in the Orbitrap or in the ion injection optics can be conducted by performing selective ion ejection of either one of two populations and then comparing the results obtained before and after ion ejection. Selective ion ejection is particularly intriguing because it might enhance exact mass measurement of ions of interest, especially of multiply charged ions, by ejecting adjacent ion species. Because ions with similar mass/charge ratios have similar rotational radii in the Orbitrap, as well as very similar axial frequencies, they spend more time together in closer proximity and thus maximize space charge effects.

Ac de-excitation and re-excitation may be useful for improving the peak shape, resolution, and mass measurement accuracy when the ion signal is distorted by space charge effects in the Orbitrap or in the injection optics. It might also be useful in studying ion packet dephasing processes in the Orbitrap and, therefore, in developing a method to extend the transient time and improve performance. Axial de-excitation, "storage" of ions at $z=0$ for up to $4 \mathrm{~s}$, and subsequent re-excitation were recently ${ }^{\circ}$ demonstrated $[8]$. This $^{\circ}$ capability is useful if long trapping of ions is desired and offers the opportunity to achieve tandem mass spectrometry in the Orbitrap.

Selective ion ejection in the Orbitrap was previously

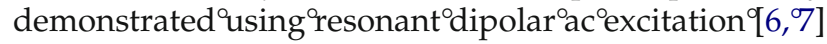
but mass resolution of ion ejection was limited by the frequency resolution of the excitation waveform and 
off-resonance excitation, the same situation as in FTICR [9]. In ${ }^{\circ}$ the ${ }^{\circ}$ Orbitrap, ${ }^{\circ}$ the ${ }^{\circ}$ highest $^{\circ}{ }^{2}$ ass $^{\circ}$ resolution ${ }^{\circ}$ for ${ }^{\circ}$ ion ejection reported was 1960 by using an excitation waveform $^{\circ}$ with $^{\circ} \mathrm{a}^{\circ}$ very $^{\circ} \operatorname{low}^{\circ}$ amplitude ${ }^{\circ}$ of $^{\circ} 0.16^{\circ} \mathrm{V}^{\circ}[6] .^{\circ} \mathrm{In}^{\circ}$ this paper, we report on a new strategy-phase-enhanced selective ion ejection-for improving mass resolution in ion ejection while using broadband ac waveforms.

\section{Experimental}

\section{Reagents}

All chemicals used were purchased from Sigma (St. Louis, MO, USA) and dissolved in water/methanol (50:50 vol \%:vol \%) with $1 \%$ (vol \%) formic acid. The sample solution contained $0.03 \mathrm{mg} / \mathrm{mL}\left[\mathrm{Val}^{5}\right]$-angiotensin II and $0.1 \mathrm{mg} / \mathrm{ml}$ Lys-des-Arg ${ }^{9}$-bradykinin. The sample was introduced into the electrospray source at a flow rate of $0.2 \mu \mathrm{L} / \mathrm{min}$ using a syringe pump (Harvard, South Natick, MA). Typically, the ESI capillary was held at $3000 \mathrm{~V}$ and room temperature.

\section{Orbitrap Mass Spectrometer}

The Orbitrap mass spectrometer used in these experiments is a prototype of the commercial Thermo Orbitrap $^{\circ}$ and $^{\circ}{ }^{\circ} s^{\circ}$ described $^{\circ}$ in $^{\circ}$ detail $^{\circ}$ elsewhere $^{\circ}[3] .{ }^{\circ}$ An $^{\circ}$ electrospray source creates ions, which are accumulated (100-400 ms), energized (1300 V), and bunched into a small ion packet with a spatial spread of a few millimeters. The ion packet is then accelerated and deflected into the Orbitrap for mass analysis.

Resonant ac waveforms are applied on the split outer electrode before ion detection. A digital delay generator (DDG, Stanford Research Systems Model DG535) triggers the waveform generator (model AFG320, Sony Tektronix); the DDG itself is activated by the ion injection gate. Therefore, the relative phase angle of the ac with respect to the ion axial motion is simply controlled ${ }^{\circ}$ by $^{\circ}$ the $^{\circ}$ delay $^{\circ}$ of $^{\circ}$ the $^{\circ} \mathrm{DDG}^{\circ}[6] .{ }^{\circ}$ Typically, $^{2}$, axial excitation was performed after an initial delay $(60-80 \mathrm{~ms})$ relative to ion injection and ion detection started $20 \mathrm{~ms}$ after ac application. The initial delay is needed for radial and angular dephasing of the ion packets $^{\circ}\left[3,{ }^{\circ} 5,{ }^{\circ} 10\right]$. Signal $^{\circ}$ is $^{\circ}$ acquired $^{\circ}$ as $^{\circ}$ an $^{\circ}$ image current and the transient is recorded for $800 \mathrm{~ms}$ at a sampling rate of $5000 \mathrm{kHz}$. The transient is Fourier transformed using the MIDAS data analysis program $[11]^{\circ}$ into $^{\circ} \mathrm{a}^{\circ}$ mass $^{\circ}$ spectrum, $^{\circ}$ with $^{\circ}$ one $^{\circ}$ level $^{\circ}$ of $^{\circ}$ zero filling and no apodization.

\section{Results and Discussion}

Mass resolution achieved in ion selection in the Orbitrap can overcome the constraints imposed by the limited frequency resolution of the applied ac waveforms. The reason for this is that the two processes of ion de-excitation/re-excitation and ion ejection (that is, ion excitation from large axial amplitude) are imple-
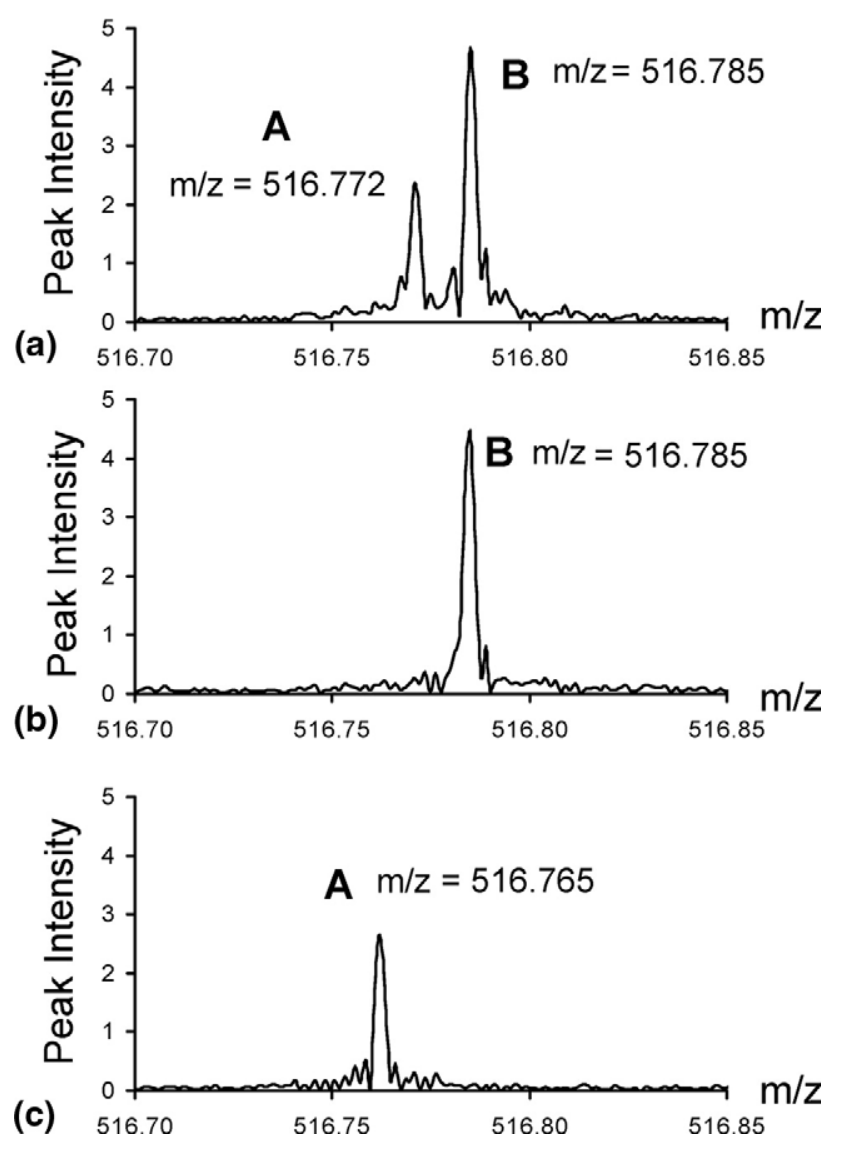

Figure 1. (a) Mass spectrum of two ions, with theoretical massto-charge ratios of $[\mathrm{M}+2 \mathrm{H}]^{2+}=516.76,671 \mathrm{Th}$ (ion $\mathrm{A}$ ) and $[\mathrm{M}+2 \mathrm{H}]^{2+}=516.78,490$ Th (ion B). (b) Mass spectrum obtained by re-exciting ion B while ejecting ion A. (c) Mass spectrum obtained by re-exciting ion $\mathrm{A}$ while ejecting ion $\mathrm{B}$.

mented using different phase relationships relative to ion axial'motion. Figure $1{ }^{\circ}$ shows $^{\circ}{ }^{\circ}{ }^{\circ} \operatorname{example}^{\circ}$ of ${ }^{\circ}$ selective ejection ${ }^{\circ}$ for ${ }^{\circ}$ ion ${ }^{\circ}$ isolation ${ }^{\circ}{ }^{\circ}{ }^{\circ}$ the $^{\circ}$ Orbitrap. ${ }^{\circ}$ Figure ${ }^{\circ} 1 a^{\circ}$ is ${ }^{\circ} a$ mass spectrum acquired by electrospraying a solution containing both $\left[\mathrm{Val}^{5}\right]$-angiotensin II and Lysdes-Arg'-bradykinin. The two doubly charged ions generated from electrospray ionization (ESI) were $[\mathrm{M}+2 \mathrm{H}]^{2+}=516.76,671 \mathrm{Th}$ (Thomson, ion A) and $[\mathrm{M}+2 \mathrm{H}]^{2+}=516.78,490 \mathrm{Th}$ (ion B). The theoretical mass/charge ratio difference between these two ions is $18.2 \mathrm{mTh}$ and the minimum mass resolution of ion selection required here is 28,400 .

The corresponding frequency spectrum of these two ions is shown in Figure $2 a$. The measured ${ }^{\circ}$ frequencies of these two ions in the Orbitrap were 485.159 and 485.168 $\mathrm{kHz}$. The ac signal used for this experiment, shown in Figure $2 \mathrm{~b}$, had ${ }^{\circ}$ an ${ }^{\circ}$ mplitude ${ }^{\circ}{ }^{\circ} 3.8^{\circ} \mathrm{V}_{\text {pp }}$. In all, 150 cycles were needed to eject ions when the waveform was applied in phase with the ion motion and 470 cycles were needed to obtain fully re-excited signals while the ac was applied $180^{\circ}$ out of phase with respect to the ion motion. Centered at a frequency of $485.16 \mathrm{kHz}$, the bandwidth of this excitation waveform is $2 \mathrm{kHz}$ (width of the main lobe at baseline) and thus can be used to 

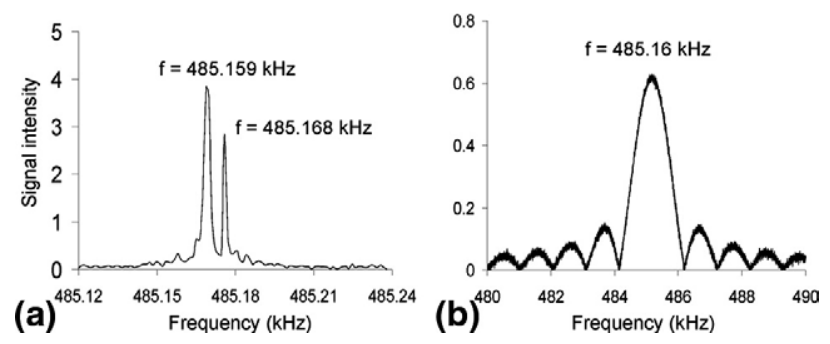

Figure 2. Two frequency spectra. (a) Frequency spectrum of two ions A and B from the Orbitrap. (b) Bandwidth of 470 cycles of ac waveform with an amplitude of $3.8 \mathrm{~V}$, which is wide enough to cover the resonant frequency range of both ions $\mathrm{A}$ and $\mathrm{B}$. Note the different frequency scales of the two plots.

excite ions at both mass-to-charge ratios. Note that Figure $2 \mathrm{a}^{\circ}{ }^{\circ}{ }^{\circ}{ }^{\circ}$ Figure $2 \mathrm{~b}$ \% ${ }^{\circ}$ ave ${ }^{\circ}$ different ${ }^{\circ}$ frequency ${ }^{\circ}$ scales.

This ac waveform was applied after specifying a delay relative to ion injection. The delay of the excitation waveform was adjusted for two different cases. In case (i), the delay was adjusted so that after the application of the ac waveform, only ion B was observed and ion $^{\circ} \mathrm{A}^{\circ}$ signal $^{\circ}$ disappeared ${ }^{\circ}{ }^{\circ}{ }^{\circ}$ shown ${ }^{\circ}{ }^{\circ}{ }^{\circ}$ Figure $1 \mathrm{~b}$. In $^{\circ}$ case (ii), the excitation ac waveform was applied with a delay of $1.05 \mu$ s longer than the delay in case (i). For case (ii), only ion A signal was detected while ion B was gone, ${ }^{\circ}{ }^{\circ}{ }^{\circ}$ hown ${ }^{\circ}{ }^{\circ}{ }^{\circ}$ Figure $^{\circ} 1 \mathrm{c}$.

In case (i), ion $B$ was de-excited and re-excited by applying the ac $180^{\circ}$ out of phase with respect to the ion motion of B. In contrast to case (i), ion A was de-excited and re-excited in case (ii) by using the ac at a phase angle of $180^{\circ}$ with respect to the ion motion of A. Note that the period of harmonic motion for both ions was $2.1 \mu \mathrm{s}$ and the difference between the two excitation delays was $1.05 \mu \mathrm{s}$ - the half-period of their harmonic motion. This means that these two excitation waveforms were applied $180^{\circ}$ out of phase with each other. Therefore, the excitation ac waveform in case (i) was applied in phase with ion A while it was $180^{\circ}$ out of phase with ion population B; and the excitation waveform in case (ii) was in phase with ion B but was $180^{\circ}$ out of phase with ion A. Because the initial ion axial amplitude in the Orbitrap is sufficiently great that excitation and ejection occur more quickly than deexcitation $[6],{ }^{\circ} 470^{\circ}$ cycles $^{\circ}$ of ${ }^{\circ}$ this ${ }^{\circ}$ ac $^{\circ}$ signal $^{\circ}$ are ${ }^{\circ}$ more ${ }^{\circ}$ than enough to eject the ion if this ion is in phase with the ac excitation signal. Therefore ion A was ejected in case (i) with ion $B$ fully re-excited and ion $B$ was ejected in case (ii) with ion $\mathrm{A}$ re-excited. The mass resolution of ion isolation for these two cases is 28,400 .

Finding this particular phase point was accomplished by simply estimating from the frequencies of the two ions. These two ions used had similar mass/ charge ratios and thus they arrived at the entrance of the Orbitrap and started their oscillations in the Orbitrap at essentially the same time. Therefore, they must have oscillated nearly in phase with each other at the beginning ${ }^{\circ}$ of $^{\circ}$ the ${ }^{\circ}$ experiment. ${ }^{\circ}$ Figure ${ }^{\circ} 3 a^{\circ}$ illustrates ${ }^{\circ}$ the relative phase relationship of these two ions right after ion injection. The beat frequency of their oscillations was about $6.9 \mathrm{~Hz}$, which is the difference of their two frequencies. This beat frequency indicates that these two groups of ions would repeatedly come into phase with each other with a period of about $144.6 \mathrm{~ms}$. Therefore, about $72.3 \mathrm{~ms}$ after ion injection, these two groups of ions would be $180^{\circ}$ out of phase with each other, ${ }^{\circ} a^{\circ}{ }^{\circ}$ shown ${ }^{\circ}$ in ${ }^{\circ}$ Figure $^{\circ} 3 b^{\circ} .^{\circ}$ It $^{\circ}$ is ${ }^{\circ}$ precisely ${ }^{\circ}$ this ${ }^{\circ}$ phase difference between the two mass-to-charge ratios-and spatial separation-that underlies the success of this method. If the ac excitation waveform was applied after an initial delay of about $72.3 \mathrm{~ms}$ after ion injection and also fine-tuned into phase with one of these two ions, then the excitation waveform would be $180^{\circ}$ out of phase with the other ion. Therefore, one ion would be ejected from the Orbitrap while the other one would be de-excited and re-excited and, thus, retained in the Orbitrap.

Interestingly, the maximum mass resolution of ion selection by this method in the Orbitrap may be estimated. In practice, the maximum mass resolution of ion selection using our method of phase-enhanced selective ion ejection is limited by the exponential decay of the time-domain transient signal obtained from the Orbitrap. The application of the ac waveform should be performed before the transient has significantly decayed; selective ejection may be achieved if the two groups of ions become $180^{\circ}$ out of phase with each other more quickly than the signal transient decays. For example, if the ion signal decays with lifetime $\tau \simeq 200$ ms (reasonable because the transient normally lasts $\left.1-1.5^{\circ} \mathrm{s}\right)^{\circ}\left[5,{ }^{\circ} 10\right]^{\circ}$, the $^{\circ}$ longest $^{\circ}$ delay $^{\circ}$ in $^{\circ}$ applying ${ }^{\circ}$ the waveform would also be $200 \mathrm{~ms}$. This is equal to the time when the two ions, close in mass, are $180^{\circ}$ out of phase with each other, or one-half of a beat period has elapsed. Therefore, the beat period of these two ions could be $2 \times 200 \mathrm{~ms}$ and the beat frequency $2.5 \mathrm{~Hz}$. For ions having mass/charge ratios around $517 \mathrm{Th}$, the maximum frequency resolution would be $R_{\mathrm{f}}=485,000$ / $2.5=194,000$, and thereby the maximum mass resolution $\mathrm{R}_{\mathrm{m}^{\circ}}={ }^{\circ} \mathrm{R}_{\mathrm{f}} / 2^{\circ}={ }^{\circ} 97,000^{\circ}[5]$.

Another interesting aspect of this experiment is that the ${ }^{\circ}$ two ${ }^{\circ}$ peaks ${ }^{\circ}$ in $^{\circ}$ Figure $^{\circ} 1 a^{\circ}$ have $^{\circ}$ an $^{\circ}$ observed $^{\circ}$ mass / charge ratio difference of $13 \mathrm{mTh}$, almost $33 \%$ less than
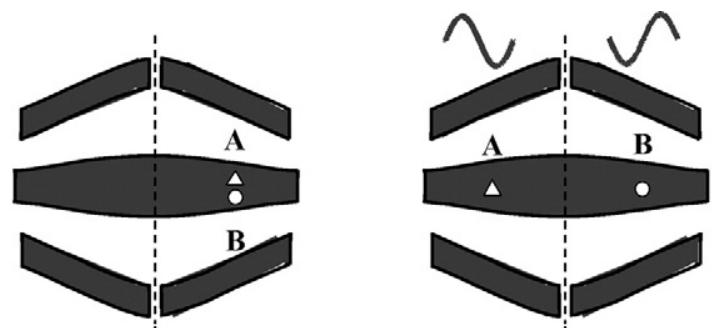

$\begin{array}{ll}\text { (a) } 0 \mathrm{~ms} \text { after ion injection (b) } 72.3 \mathrm{~ms} \text { after ion injection } & \end{array}$

Figure 3. Relative phase relationship of two ions A and B. (a) These two ions are in phase with each other right after ion injection. (b) These two ions are $180^{\circ}$ out of phase with each other $72.3 \mathrm{~ms}$ after ion injection. 
the theoretical value of $18.2 \mathrm{mTh}$. After ion A was ejected $^{\circ}\left(\text { as }^{\circ} \text { shown }^{\circ} \text { in }^{\circ} \text { Figure }^{\circ} 1 \mathrm{~b}\right)^{\circ}$ and $^{\circ}$ after $^{\circ}$ ion $^{\circ} \mathrm{B}^{\circ}$ was ejected $^{\circ}\left(\right.$ as $^{\circ}$ shown $^{\circ}$ in $^{\circ}$ Figure $\left.^{\circ} 1 \mathrm{c}\right),{ }^{\circ}$ the ${ }^{\circ}$ measured $^{\circ}$ mass / charge ratio difference of these two ions is $20 \mathrm{mTh}$, a more accurate value, even though this number is from two different mass spectra (these two spectra were collected one immediately after the other). The standard deviation in the observed frequency for either peak was $<0.6 \mathrm{~Hz}$, roughly 1 part per million. This corresponds to a 2 part per million standard deviation in the mass, $1 \mathrm{mTh}$.

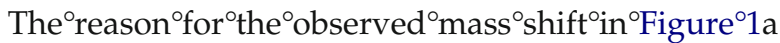
might be space charge operating in the Orbitrap. First, these ions are both doubly charged, increasing the strength of the Coulomb interaction fourfold over the interaction of two singly charged species. Second, the two ion populations spend considerable time in close proximity. Because they have nearly identical axial frequencies, the ions will stay in phase much longer than, say, two ions separated by even one mass unit. Additionally, because the two ions have very similar mass/charge ratios, they will have almost the same rotational radius, placing the angularly and radially dephased "rings" of ions in close contact. After the ejection of either one of the two ions, the space charge effect is reduced. More experiments will be conducted; for example, an internal mass (frequency) standard can be added to provide solid evidence for the mass shift of the two ions in the normal mass spectrum and then measure the mass accuracy after the ejection of either ion.

\section{Acknowledgments}

This work was funded by the National Science Foundation, Grant CHE-0216239 (Major Research Instrumentation Program), the Office of Naval Research (ONR) N00014-02-0834, and Thermo Electron Corporation. We thank Dr. Brian Laughlin for creating the sketch $^{\circ}$ of $^{\circ}$ the ${ }^{\circ}$ orbitrap $^{\circ}$ electrodes ${ }^{\circ}$ in $^{\circ}$ Figure 3 .

\section{References}

1. Busch, K. L.; Glish, G. L.; McLuckey, S. A. Mass Spectrometry/Mass Spectrometry: Techniques and Applications of Tandem Mass Spectrometry; John Wiley \& Sons: New York; 1988.

2. Makarov, A.; Denisov, E.; Kholomeev, A.; Balschun, W.; Lange, O.; Strupat, K.; Horning, S. Performance Evaluation of a Hybrid Linear Ion Trap/Orbitrap Mass Spectrometer. Anal. Chem. 2006, 78, 2113-2120.

3. Hu, Q.; Noll, R. J.; Li, H.; Makarov, A.; Hardman, M.; Cooks, R. G. The Orbitrap: A New Mass Spectrometer. J. Mass Spectrom. 2005, 40, $430-$ 443.

4. Makarov, A.; Denisov, E.; Lange, O.; Horning, S. Dynamic Range of Mass Accuracy in LTQ Orbitrap Hybrid Mass Spectrometer. J. Am. Soc Mass Spectrom. 2006, 17, 977-982.

5. Makarov, A. Electrostatic Axially Harmonic Orbital Trapping: A Highperformance Technique of Mass Analysis. Anal. Chem. 2000, 72, 1156 1162

6. Hu, Q.; Makarov, A.; Cooks, R. G.; Noll, R. J. Resonant AC Dipolar Excitation for Ion Motion Control in the Orbitrap Mass Analyzer. J. Phys. Chem. A 2006, 110, 2682-2689.

7. Wu, G.; Noll, R. J.; Plass, W. R.; Hu, Q.; Perry, R. H.; Cooks, R. G. Ion Trajectory Simulations of Axial AC Dipolar Excitation in the Orbitrap. Int. J. Mass Spectrom. 2006, 254, 53-62

8. Noll, R. J.; Hu, Q.; Wu, G.; Plass, W.; Perry, R.; Cooks, R. G. Driving Ion Axial Motion in the Orbitrap Mass Analyzer. Proceedings of the 54th ASMS Conference on Mass Spectrometry and Allied Topics, Seattle, WA, May/June 2006; DVD-ROM.

9. Heck, A. J. R.; de Koning, L. J.; Pinkse, F. A.; Nibbering, N. M. M. Mass-Specific Selection of Ions in Fourier-Transform Ion Cyclotron Resonance Mass Spectrometry. Unintentional Off-Resonance Cyclotron Excitation of Selected Ions. Rapid Commun. Mass. Spectrom. 1991, 5 , 406-414.

10. Hardman, M.; Makarov, A. Interfacing the Orbitrap Mass Analyzer to an Electrospray Ion Source. Anal. Chem. 2003, 75, 1699-1705.

11. Senko, M.; Canterbury, J.; Guan, S.; Marshall, A. A High-Performance Modular Data System for Fourier Transform Ion Cyclotron Resonance Mass Spectrometry. Rapid Commun. Mass Spectrom. 1996, 10, 1839-1844. 